\title{
Cloud Computing As an Emerging Paradigm in E-Learning: SWOT Analysis
}

\author{
'JIT, Borawan, MP, India, \\ tak_reena@rediff.com \\ 2JIT, Borawan, MP, India, \\ dr_atul1@yahoo.com
}

Dr. Reena M. Tak ${ }^{1}$, Dr. Atul Upadhyay ${ }^{2}$

\begin{abstract}
E-learning, also known as CBT (Computer-Based Training), IBT (Internet-Based Training) or WBT (Web-Based Training), refers to electronic learning where electronic devices like computer and technology like internet and its services play an important role in teaching and learning process. The content is delivered digitally with learning support and other services to the learner. To successfully implement this process, hi-tech devices and technological advancements need to be accommodated in due course. Huge investments in establishing all these resources became a bottleneck for medium and small scale institutions in India. Cloud computing emerged as a solution as it provide almost any service as a shared and pay per use resource. It provides resources and capabilities of information technology via services offered by cloud service provider through internet. In this paper, the authors study the e-learning scenario and cloud computing as a solution provider. The authors also did a quick Strength, Weakness, opportunity, Threat (SWOT) analysis for the above mentioned context.
\end{abstract}

\section{INDEX TERMS}

cloud computing, e-learning, Infrastructure, Platform, Security, Software, SWOT analysis, virtualization

\section{Council for Innovative Research}

Peer Review Research Publishing System

Journal: International Journal of Management \& Information Technology

Vol. 7, No. 2

editor@cirworld.com

www.cirworld.com, member.cirworld.com 


\section{INTRODUCTION}

Education is in a constant state of evolution throughout the globe. The main idea behind this lies in adoption of revolutionary changes and trends in education, pedagogy, technology and teaching learning environment. A new generation of students is emerging very fast who are either ready or becoming ready to face the challenges of $21 \mathrm{st}$ century. The education technology is changing and shifting its paradigm from traditional didactic pedagogy of teacher centered approach to student-centered constructivists' approaches whereby students construct knowledge through interaction and collaboration with peers as well as teachers. The bulk of today's e-learning systems still consist of simple conversion of classroom-based content to an electronic format while still retaining its traditional distinctive knowledgecentric nature (Teo et al. 2006).

The emerging technologies play an important role in the construction of new pedagogies, where control can shift from teacher to autonomous learner who can build his own domain of knowledge generation and dissemination into his own group and community of friends and co-learners. It is evident that traditional methods of teaching and learning process are unable to foster the needs and demands of a changing knowledge and communication-based society. The rapid advances in technology in the last few decades have had a significant impact on work, leisure, culture and social interaction. The kind of skills students need to develop to be prepared for the 21 st century is different from what they needed the previous century.

\section{Evolution of E-Learning}

E-learning services have evolved since computers were first used in education. There is a trend to move towards blended learning services, where computer-based activities are integrated with practical or classroom-based situation. E-learning refers to the use of electronic media and information and communication technologies (ICT) in education. E-learning is broadly inclusive of all forms of educational technology in learning and teaching. E-learning is inclusive of, and is broadly synonymous with multimedia learning, technology-enhanced learning (TEL), computer-based instruction (CBI), computerbased training (CBT), computer-assisted instruction or computer-aided instruction (CAI), internet-based training (IBT), web-based training (WBT), online education, virtual education, virtual learning environments (VLE) (which are also called learning platforms), m-learning, and digital educational collaboration. These alternative names emphasize a particular aspect, component or delivery method.

Depending upon the major technology used, e-learning can be broadly classified as:

2.1 Web-Based Learning Web-based training (sometimes called e-learning) is anywhere, any-time instruction delivered over the Internet or a corporate intranet to browser-equipped learners. There are two primary models of Webbased instruction: synchronous (instructor-facilitated) and asynchronous (self-directed, self-paced). Instruction can be delivered by a combination of static methods (learning portals, hyperlinked pages, screen cam tutorials, streaming audio/video, and live Web broadcasts) and interactive methods (threaded discussions, chats, and desk-top video conferencing).

2.2Computer-Based Training Computer-Based Training (CBT) is an interactive method of learning that provides a series of self-paced, hands-on, web-based courses. CBT offers technology, managerial, and supervisory curricula. Acronym for computer-based training, a type of education in which the student learns by executing special training programs on a computer. CBT is especially effective for training people to use computer applications because the CBT program can be integrated with the applications so that students can practice using the application as they learn.

2.3Virtual Education Opportunities The terms virtual learning environment (VLE) and learning platform are generically used to describe a range of integrated web based applications that provide teachers, learners, parents and others involved in education with information, tools and resources to support and enhance educational delivery and management. These terms are broadly synonymous with 'managed learning environments' (MLEs) and 'managed virtual learning environments' (MVLEs).

The applications that form part of these online services can include web pages, email, message boards and discussion forums, text and video conferencing, shared diaries, online social areas, as well as assessment, management and tracking tools.

2.4 Digital Collaboration Computer (CSCL) uses instructional methods designed to encourage or require students to work together on learning tasks. CSCL is similar in concept to the terminology, "e-learning 2.0".

Collaborative learning is distinguishable from the traditional approach to instruction in which the instructor is the principal source of knowledge and skills. For example, the neologism "e-learning 1.0" refers to the direct transfer method in computer-based learning and training systems (CBL). In contrast to the linear delivery of content, often directly from the instructor's material, CSCL uses blogs, wikis, and cloud-based document portals (such as Google Docs and Dropbox). With technological Web 2.0 advances, sharing information between multiple people in a network has become much easier and use has increased. One of the main reasons for its usage states that it is "a breeding ground for creative and engaging educational endeavors."

Using Web 2.0 social tools in the classroom allows for students and teachers to work collaboratively, discuss ideas, and promote information. Blogs, wikis, and social networking skills are found to be significantly useful in the classroom. After 
initial instruction on using the tools, students also reported an increase in knowledge and comfort level for using Web 2.0 tools. The collaborative tools also prepare students with technology skills necessary in today's workforce.

Locus of control remains an important consideration in successful engagement of e-learners. The continuing attention to aspects of motivation and success in regard to e-learning should be kept in context and concert with other educational efforts. Information about motivational tendencies can help educators, psychologists, and technologists develop insights to help students perform better academically.

Cloud Computing appeared as a global solution to the requirements and demands of arena of e-learning. Various educational institutions recognize that adopting the latest technologies and solutions is essential to stay competitive and retaining students. Cloud computing can actually help institutions reduce high expenditures on hardware, software and IT maintenance. Cloud computing provides institutions with a centralized, virtual data center that is accessible to faculty and admissions personnel, for example, at any time and any location. "Clouds are a large pool of easily usable and accessible virtualized resources (such as hardware, development platforms and/or services). These resources can be dynamically reconfigured to adjust to a variable load (scale), allowing also for an optimum resource utilization. The pool of resources is typically exploited by a pay-per-use model where guarantees are offered by the infrastructure Provider by means of customized SLAs" [1].

Cloud Computing in the e-learning arena is becoming popular among e-learners because of its Greater connectivity between centralized server-side applications and low cost/low processor capacity mobile devices (M-learning).This results in better access, more control, and greater freedom due to greater cost savings from scalable architectures and open source products, and the possibility of higher learning outcomes.[2]

However, some serious issues with the viability and suitability of the Cloud Computing model for education remain: primarily, storage of sensitive data on third-party servers outside of the organization. Family and individual privacy are important. Also the loss of data through discontinued Cloud services has not been uncommon.

\section{STRATEGIC FRAMEWORKS FOR CLOUD MIGRATION IN E-LEARNING SYSTEM}

Cloud computing offers several services which can be broken down into 3 major categories:

\subsection{IAAS: Hardware Layer}

Infrastructure as a Service comprises the layer of storage, hardware, servers and networking components. The supplier maintains and upgrades these resources and the user pays for the service depending on the amount of usage. The major advantage is that users only pay for the exact amount of resources used, and resources available can be easily scaled to accommodate rapidly changing needs. Architecture scalability is achieved through Full- or Para-virtualization, such that multiple systems or operating systems can be run at the same time on a virtual machine or across multiple machines.

\subsection{PAAS: Platform Layer}

Platform as a Service can also be supplied by the Cloud and allows software and service development without downloading tools and software to client machines. Using the Cloud, large and complicated software packages can be developed, tested and disseminated, again leveraging the benefits of virtualization and scalability.

\subsection{SAAS: Application Layer}

Software as a Service is one of the most common uses of Cloud Computing, exemplified by Google's Gmail. Clients access software services such as email, word processing, spreadsheets, etc. from the Cloud instead of running these applications directly on their client computers.

"Unlike a mainframe, which is a physical machine that offers finite computing power, a cloud represents all possible resources on the Internet, suggesting infinite power and capacity. Meanwhile, unlike a simple terminal acting as a user interface to a mainframe, a PC in the cloud computing paradigm possesses significant power to provide a certain degree of local computing and caching support" [3].

\section{PRACTICAL APPLICATION OF CLOUD COMPUTING IN E-LEARNING}

Cloud concept has significant implications as a communication medium. While it may not be highly interactive in a physical sense, it has strong potential for social interactivity. The goal of utilizing this type of tool is the achievement of 'virtual communities' of educators, researchers and practitioners on the Internet working in small collaborative groups which may help to promote a more reflective meta cognitive approach in tackling problems and advancing the practices. The cloud computing can be used for education purpose in more than one ways. It can be used effectively for content creation, content delivery, education platform, teaching evaluation and education management.

In order to succeed in research projects and to develop quality work through iterative processes, the role of active collaboration with colleagues and experts in the field at its various stages of development cannot be over looked.

The 'cloud computing' provides an easy user-friendly environment / platform for this type of collaboration. The cloud platform can support teachers to prepare teaching portfolio; presentation on teaching to a local audience; a conference presentation; a manuscript to be submitted for publication, etc. It may also include, for the purpose of critical review and evaluation, self-reported e-Portfolios that summarizes a teacher's major teaching accomplishments and strengths in the form of short descriptions of activities and achievements (e.g., what and how they teach-types of instructional methods, 
materials, and techniques, why they teach that way, and whether or not it works with evidences), feedback from peers based on teaching observation and peer review of related scholarly activities, feedback from students based on their views on instructional activities, and the end-of-course student evaluation instrument.

Several private Cloud e-learning systems have been developed and documented:

- BlueSky Cloud framework at Xian Jiaotong University in China enables virtualization of physical machines to be allocated on the fly and "delivers reliable, scalable and cost-efficient services to E-Learning systems" [4].

- "The Hochschule Furtwangen University (HFU) is running their own private cloud infrastructure, called Cloud Infrastructure and Application CloudIA. The targeted users of the CloudIA project are HFU staffand students running e-Learning applications, and external people for collaboration purposes" [5]

- $\quad$ Major projects are further underway in Japan and Malaysia [6][7].

- A Google Docs-like portal running on Amazon Elastic Cloud (EC2) for teaching statistics using R and Scilab software has been successful in teaching mathematics and statistics [8].

\section{BENEFITS}

As Grossman points out, "Cloud computing is often offered with a pricing model that lets you pay as you go and for just the services that you need. For example, if you need an additional 1,000 computing instances for an hour, you pay just for these 1,000 computing instances and just for the hour that you use them. No capital expenditure is required" [9]. Price is also mentioned as a positive feature of adopting the Cloud for learning in Zhang Guoli and Liu Wanjun's survey of Cloud Computing in the development of platform architecture [10].

Overall, not just hardware costs, but also system management, licensing costs and fees can be reduced as users only pay for the software they need (concept of on-demand software). User agreements and contracts can be simplified, with the user only paying for individual software instances and also avoiding the time and cost of patches and updates. As Monfort observes, "SaaS software vendors may host the application on their own web servers or download the application to the consumer device, disabling it after use or after the "on-demand" contract expires. "On-demand" licensing and use alleviates the customer's burden of equipping a device with every conceivable application. It also reduces traditional End User License Agreement (EULA) software maintenance, ongoing operation patches, and patch support complexity in an organization" [11].

For academic purposes, cloud computing can be very functional. This technology can be used in a very constructive manner. Cloud can be used in e-learning in number of ways as follows:

- This provides opportunity for ubiquitous computing for those who are not available during regular time.

- Cloud provides data and database as a service, wherein personal data can be used and stored by individual as a personal workspace.

- Cloud can be proven as a convenient tool to fit into place in the scholarship of teaching and learning

- Personal Learning Environments (PLEs) used by many people as an alternative to institutionally controlled Virtual Learning Environments (VLEs)/LMS with different personalized tools to meet their own personal needs and preferences, because teaching and learning is a constant process.

- $\quad$ No need for backing up everything to a thumb drive and transferring it from one device to another.

- No need to copy all stuff from one PC to another when buying a new one. It also means you can create a repository of information that stays with you and keeps growing as long as you want.

\section{CHALLENGES WITH CLOUD COMPUTING}

\subsection{Security \& Privacy Concerns}

Miller echoes an often-repeated concern about Cloud Computing privacy limitations: "Providers, users, and the federal government agree almost universally that cyber security is the predominant risk" [12]. A survey of 147 educators and endusers working in technology-enhanced learning environments in 17 countries by German and Slovenian researchers demonstrated that the "Majority of the respondents are not satisfied with the current level of data protection and privacy in TEL, and they believe that the related problems will increase in the next 5 years" [13]. Cloud computing may introduce additional security and privacy concerns, although the topic is controversial in this regard. Privacy is closely related to security, and thus far only $2 \%$ of European businesses have adopted Infrastructure as a Service (laaS) according to the analyst company Forrester Research, due to privacy concerns [14].

Pocatilu [15], on the other hand, believes that Cloud Computing can be inherently more secure than current architectures. Although counterintuitive, the Cloud according to Pocatilu provides security benefits: improved improbability, since it is nearly impossible for a thief to locate a virtualized machine, and virtualization allows the rapid replacement of a compromised machine; centralized data storage means that the loss of peripheral devices like laptops, PDAs are not catastrophic for a company; oversight is easier, as personnel do not have to monitor thousands of individual computers. 
However, the University of Massachusetts (UMass) introduced Google Apps for primarily email services in 2009 and then discontinued the service in 2010. Although low adoption rates among students was one reason for the cancellation, another reason was that "legitimate concerns over security" arose as UMass continued to explore the service and shortly before opening Google Apps to undergrads, the University decided not to offer it to faculty or staff as it had originally planned. Because faculty and staff are state employees and at times use e-mail to send confidential information, they must follow state and federal security standards when communicating via electronic or other means" [16]. But Google, disappointed with this decision, has countered that its Posting Services provide adequate internet security to control email addresses, provide filters for inappropriate language and cyber bullying, and encrypt messages.

In addition, "some cloud services reserve intellectual property (IP) rights over everything you post so may lose IP in critical materials or to collections of materials - such as those you compile on Delicious" [17].

Security concerns are also raised by some Cloud vendor policies of retaining deleted account information: in fact, clients are often lured into free services in exchange for the personal data they contribute to a site as well as their web activity as recorded by cookies or server logs. All of this is valuable information for marketers and laws on how this information can be used and sold are considerably murky in countries such as the U.S. As SMART Communications, a Philippines wireless network provider, warns, "Deleted accounts do not mean deleted contents. Content can be retained forever even if you wish the opposite, and even if it was your content in the first place. This is often specified in the terms and conditions of service"[18].

We believe that there are no fundamental obstacles to make a cloud-computing environment as secure as the vast majority of in-house IT environments, and that many of the obstacles can be overcome immediately with well understood technologies such as encrypted storage, Virtual Local Area Networks, and network middle boxes (e.g. firewalls, packet filters). For example, encrypting data before placing it in a Cloud may be even more secure than unencrypted data in a local data center; this approach was successfully used by TC3, a healthcare company with access to sensitive patient records and healthcare claims, when moving their HIPAA-compliant application to AWS" [19].

\subsection{Reliability Concerns}

But many free cloud services that educational institutions are looking at for adoption are in the beta phase or may be run on a non-sustainable financial plan. Millea observes: "Applications in the cloud are provided by commercial entities to make a profit: sometimes they are still in beta, sometimes they are from start-ups funded by venture capital which may run out, sometimes it is decided they are not viable. That means there is no guarantee that service you use and rely on today will continue to exist, and your transition time from one service to another may be limited" [20]. An example of a discontinued Cloud service was Google's 3D service "Lively," a response to Linden Lab's increasingly popular online virtual world Second Life. Just as with Second Life, educators began building educational modules on the site, but it abruptly closed in December, 2008 only months after it had been introduced during the summer time.

\subsection{Cultural Issues}

There are many cultural concerns with using Cloud Computing vendors which have not yet been fully investigated. In addition, there are unresolved problems with e-learning itself, as surveyed by Weber in 2011 [21]. What if, for example, an external site is supported by advertising with content inappropriate to the cultural relevance of a country, such as dating websites? Inappropriate content, especially if accessed by children, has been widely cited by Islamic officials involved in elearning, e-commerce, and e-government as a major barrier for wider adoption of the internet in the Islamic region. But the perception that content on the internet could undermine or weaken traditional values is widespread.

\subsection{Flexibility, Independent Control of Learning Objects}

The introduction of the personal computer (PC) in the 1970s was hailed as liberation from centralized computer systems (mainframes) controlled by administrators. The development of learning objects and e-learning systems has certainly been facilitated since the mid-1990s by the flexibility and control inherent in controlling one's own hardware and software, particularly when one thinks of Linux-based or open source products that have recently emerged such as Moodle. Despite the many potential benefits of Cloud Computing, Jonathan Weber points out that there is a "flip side, though, is that you're dependent on someone else for your technology, and that can limit your flexibility and even your creativity. In fact, the personal computer itself arose because the old model of business computing, in which companies had big mainframes (a kind of a cloud) and everyone connected to them via "dumb" terminals, was enormously frustrating for the people sitting at those dumb terminals. They could only do what they were authorized to do. They were dependent on the computer administrators to give them permission or fix problems. They had no way of staying up on the latest innovations. The personal computer was a rebellion against the tyranny of centralized computing operations - and of the IBM mainframe world in particular [22]. The increasing ubiquity of Cloud services, in fact, may be somewhat more sinister than proponents of Cloud Computing for e-learning are willing to admit. Weber continues: "over the long run the lack of flexibility inherent in someone else running your tech will become a problem again. You'll be able to get any kind of software or service you like for a very low price - as long as it's a piece of software or service that Google and IBM think is appropriate" [23].

\section{SWOT ANALYSIS: FOR CLOUD IN E-LEARNING}

SWOT analysis (alternatively SWOT Matrix), a management term, is a structured planning method used to evaluate the Strengths, Weaknesses, Opportunities, and Threats involved in a project or in a business venture. It involves specifying the objective of the business venture or project and identifying the internal and external factors that are favorable and unfavorable to achieve that objective. 
Strengths: characteristics of the business or project that give it an advantage over others

Weaknesses: are characteristics that place the team at a disadvantage relative to others

Opportunities: elements that the project could exploit to its advantage

Threats: elements in the environment that could cause trouble for the business or project

\subsection{Strengths}

After having a complete analysis of what e-learning is and how cloud computing can be correlated to it, we are now in a position to check out the scenario of e-learning where cloud computing can be implemented. Following are the strengths of this implementation:

- When identifying the needs of the learners and knowledge providers whether it is government agencies or civil society or academicians, we come to a conclusion that both knowledge seeker and knowledge provider can collaborate through a web based network.

- $\quad$ Cloud computing is based on the idea of developing network where cooperation through collaboration is done in a cost efficient way without investing much on services and IT resources. A national knowledge network could be formed where every entity of the teaching learning process can participate and collaborate.

- With the advancements of technology especially the ICT tools like computer and communication tools like mobile etc. can enlarge the penetration of technology in society and thus results in greater awareness for internet usage. Consequently, services over the internet can be exploited for the benefit of teaching learning process.

- The usage of technology can be benefitted by even small and medium enterprises through evolving trends like optical fibers and satellite communication provided by government agencies.

\subsection{Weaknesses}

Cloud computing is although an emerging paradigm in IT industry which is expanding in all the areas but still there are hurdles in wide acceptance of it. Following are some of the internal factors which are acting as weaknesses for implementation of cloud computing in e-learning scenario:

- In developing countries, especially in India, the growth rate for adoption of IPv6 is very low. The IPv6 is responsible for fast access to internet services in a smooth way. When newer technologies are not available, then it becomes difficult to migrate to emerging paradigms like cloud computing.

- In India, the IT industry is focusing more on exporting the services to foreign countries than working on rural sections of the country. With this scenario, most of the population of India seems to be deprived of the facilities even though we see wide acceptance and availability in urban areas of the nation.

- Cloud computing is based on the idea of virtualization, and this concept is not so easy to implement. Big giants of the IT industry are providing these services but not small ones at local level.

- It is difficult to upgrade new technologies. Users and vendors need to consider several issues before transforming their system into new ones.

- When education is provided by government, the entire system is involved which comes through bureaucratic layers. It is not so easy to put into operation new work culture by converting the whole system. Some government agencies rely on Private companies of IT industry to get advanced services.

\subsection{Opportunities}

It is not so difficult to identify the areas of new opportunities while working with cloud computing analysis in elearning scenarios. We can also have a look on some external factors which might lead to availing new vistas for e-learning with cloud computing.

- The global scenario is changing and to achieve world class standard of education, we should go for trends and technologies of international acceptance. This is definitely going to be a boost up factor for change in education system.

- Government is not only promoting education through ICT but it is also looking for betterment of it through employing promising and upcoming drift and trends of technology. National mission on education through ICT is one such initiative.

- To be able to use e-learning at different levels of education, government is providing not only resources for internet but also modifying the education system for primary levels.

\subsection{Threats}

There are certain factors which may lead to slow adoption of new technology in current scenario of education system. 
- Low adoption of new emerging technologies like Internet Protocol version 6 may decelerate the process of service adoption provided by cloud computing.

- When moving towards new environment of teaching and learning process, it is difficult to identify good trainers who themselves are good at learning and adopting new methodologies and then inculcate these to their students. If there is an incessant lack of such trainers then this state may lead to decline in e-learning services and global share in this scenario.

- In India and other developing countries, vernacular languages are more popular than global languages like English, in which most of the research and development work is going on. This situation also leads to low adoption of teaching and learning materials available throughout the globe.

\section{CONCLUSION}

The idea behind cloud computing is to deliver Infrastructure, Platform and Software-as-a-Service (laaS, PaaS and SaaS) over the Internet on an easy pay-per-use business model. Clearly, Cloud Computing holds many benefits for countries in which e-learning systems are coming to maturity. The prospect of outsourcing the time and cost involved in maintaining data centers, platforms and software for delivering e-learning objects will be particularly attractive to countries with constrained education budgets, or for areas with ICT personnel shortages and low PC penetration rates. However, security, reliability, and privacy issues have not been resolved satisfactorily even among the largest currently operating Cloud Computing vendors in which control of personal data is of paramount importance. Since cloud offerings overweighs the threats associated with it, it seems that cloud computing will revolutionize the mechanism of learning especially elearning.

\section{REFERENCES}

[1] Vaquero, L., Roderp-Merino, L., 2009, A Break in the Clouds: Towards a Cloud Definition. ACM SIGCOMM Computer Commuications Review, Vol 39 (1), Pages 50-55.

[2] Alan S. WEBER, Cloud Computing In Education In The Middle East And North Africa (Mena) Region: Can Barriers Be Overcome? The 7th international conference on e-learning and software for education, Bucharest,April 28,29 2011.

[3] Voas, J., Zhang, J. 2009, "Cloud Computing: New Wine or Just a New Bottle? IT Pro, March/April 2009, Page 16.

[4] Dong, B., Zheng, Q., Qiao, M., Shu, J. and Yang, J., 2009, BlueSky Cloud Framework: An E-Learning Framework Embracing Cloud Computing. Cloud Computing, Lecture Notes in Computer Science, 2009, Volume 5931/2009, Pages 577-582. See also the related: Dong, B. Zheng, Q, Yang, J., Li, H., Qiao, M. An E-learning Ecosystem Based on Cloud Computing Infrastructure. 2009 Ninth IEEE International Conference on Advanced Learning Technologies.

[5] Doelitzscher, F., Sulistio, A., Reich, C., Kuijs, H., and Wolf, D., 2009, Private cloud for collaboration and eLearning services: from laaS to SaaS. Mathematics and Statistics Computing 5931 (2009), Pages 583-588.

[6] Rajam, S., Cortez, R., Vazhenin, A., Bhall, S., 2010, E-Learning Computational Cloud (eLC2): Web Services Platform to Enhance Task Collaboration, 2010 IEEE/WIC/ACM International Conference on Web Intelligence and Intelligent Agent Technology, Pages 350-55.

[7] Razak, S.F.A., 2009, Cloud computing in Malaysia Universities. Innovative Technologies in Intelligent Systems and Industrial Applications, 2009. CITISIA 2009.

[8] Chine, K., 2010, Learning Math and Statistics on the Cloud: Towards an EC2-based Google Docs-like Portal for Teaching/Learning Collaboratively with R and Scilab. 2010 10th IEEE International Conference on Advanced Learning Technologies, 752-53.

[9] Grossman, R.L., 2009, The Case for Cloud Computing, IT Pro, March/April 2009, Page 24.

[10] Guoli, Z., and Wanjun, L., 2010, The Applied Research of Cloud Computing Platform Architecture In the ELearning Area, The 2nd International Conference on Computer and Automation Engineering (ICCAE), 2010, Pages 356 - 359.

[11] Monfort, V., Khemaja, M., Ammari, N., Fehli, F. Using SaaS and Cloud computing For "On Demand" E Learning Services Application to Navigation and Fishing Simulator, 2010 10th IEEE International Conference on Advanced Learning Technologies, Page 663.

[12] Miller, H.G., and J. Veiga, 2009, "Cloud Computing: Will Commodity Services Benefit Users Long Term? ITPro. 2009, Page 57.

[13] Klobucar, T., Jenabi, M., Kaibel, A., Karapidis, A., 2007, Security and privacy issues in technology-enhanced learning. In: Expanding the Knowledge Economy: Issues, Applications, Case Studies; P. Cunningham, M. Cunningham (Eds.) IOS Press, 2007 Amsterdam.

[14] Byrne, B., 2011. "Cloud Computing: What You Should and Shouldn"t be Worried About." meshIP. 4 Feb. 2011. http://meship.com/Blog/2011/02/04. 
[15] Pocatilu, P., Alecu, F., Vetrici, M., 2009, Using Cloud Computing for E-learning Systems, Recent Advances on Data Networks, Communications, Computers, Proceedings of the 8th WSEAS, Page 56.

[16] Rocheleau, M., 2011, The Massachusetts Daily Collegion, 13 March 2011.

[17] Millea, J. Heading into the cloud: cloud computing and education, Education.au, Web.http://blogs.educationau.edu.au/jmillea/2009/06/23/heading-into-the-cloud-cloud-computing-and-education/

[18] SMART Communications,

2011. http://www.smartschools.ph/SmartSchools/SmartTools/UpsDownsCloudComputing.htm

[19] Armbrust, M., et al., 2009, Above the Clouds: A Berkeley View of Cloud Computing, Electrical Engineering and Computer Sciences, University of California at Berkeley, Page 17.

[20] Millea, J. Heading into the cloud: cloud computing and education, Education.au, Web. http://blogs.educationau.edu.au/jmillea/2009/06/23/heading-into-the-cloud-cloud-computing-and-education/

[21] Weber, A.S., 2011, "Cultural Dimensions of E-learning in Islamic Countries", Proceedings of INTED2011. Valencia, Spain: IATED.

[22] Weber, Jonathan, 2009, Cloud computing: Are there dangers to having information infrastructure, software and services hosted on the internet rather than on our own personal computers? http://technology.timesonline.co.uk/tol/news/tech_and_web/article3874599.ece

[23] Weber, Jonathan, 2009, Cloud computing: Are there dangers to having information infrastructure, software and services hosted on the internet rather than on our own personal computers? http://technology.timesonline.co.uk/tol/news/tech_and_web/article3874599.ece

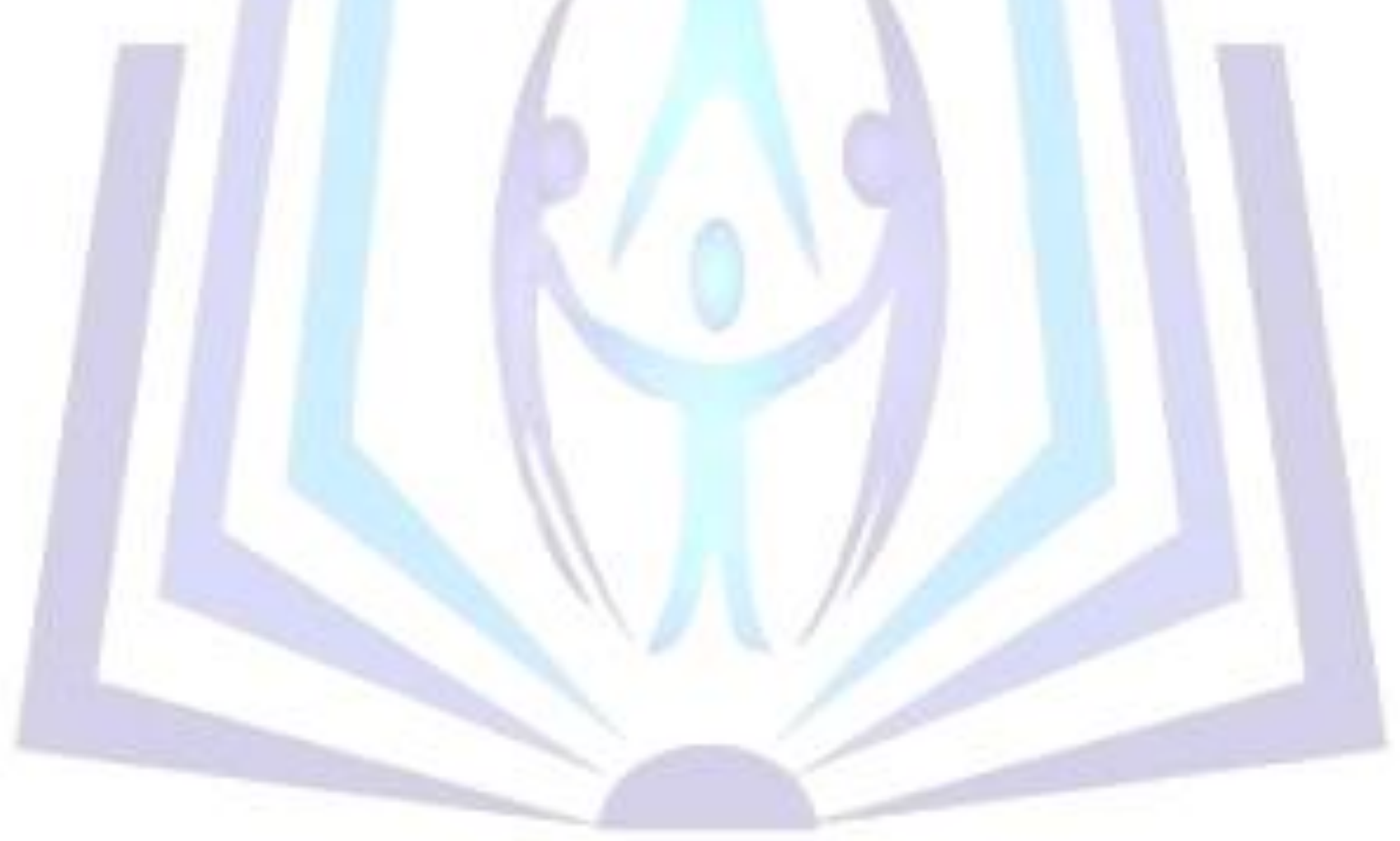

